\title{
Planejamento urbano e adaptação climática: entre possibilidades e desafios em duas grandes cidades brasileiras ${ }^{\star}$
}

\author{
Rylanneive Leonardo Pontes Teixeira** \\ Zoraide Souza Pessoa**
}

O objetivo deste artigo é investigar como as cidades de Natal e Curitiba têm incorporado a questão climática, com enfoque especial para a adaptação, em suas agendas urbanas, compreendendo como o planejamento urbano pode contribuir para a inserção da capacidade adaptativa climática local. Para isso, a metodologia apresenta uma abordagem de natureza qualitativa, utilizando as pesquisas bibliográfica, documental e de campo, com aplicação de entrevistas semiestruturadas, para o alcance dos resultados. Os dados coletados são analisados por meio da análise de conteúdo. A partir dos resultados obtidos, conclui-se que a incorporação da questão climática, sobretudo da adaptação, no planejamento e na gestão de Natal e de Curitiba ainda se apresenta como um grande e complexo desafio.

Palavras-chave: Mudanças climáticas. Capacidade adaptativa. Governos locais. Sustentabilidade urbana. Brasil.

\footnotetext{
* Este artigo é fruto da dissertação de mestrado do primeiro autor, que contou com financiamento da Coordenação de Aperfeiçoamento de Pessoal de Nível Superior (Capes).

** Universidade Federal do Rio Grande do Norte (UFRN), Natal-RN, Brasil (pontesrylanneive@gmail.com; https://orcid. org/0000-0001-8635-3234).

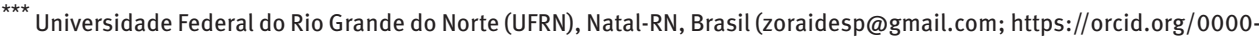
0002-9509-5027).
} 


\section{Introdução}

Ao mesmo tempo que as cidades contribuem para a intensificação das mudanças climáticas em função da alta concentração populacional nesses espaços, ${ }^{1}$ tornando-se vulneráveis e suscetíveis aos impactos climáticos, também podem exercer função protagonista no enfrentamento do problema por meio da mitigação das emissões de Gases do Efeito Estufa (GEE) e/ou da adaptação aos efeitos das mudanças climáticas (AYLETT, 2014; LECK; ROBERTS, 2015; RYAN, 2015). As cidades têm, por meio dos governos locais, papel central no planejamento alinhado às mudanças climáticas, voltado seja para mitigação ou adaptação (BROTO, 2017).

É importante destacar que, especificamente no âmbito das grandes cidades, estes espaços possuem papel crucial no enfrentamento das mudanças climáticas (HUITEMA; BOASSON; BEUNEN, 2018), uma vez que, dentre outros aspectos, apresentam maior capacidade de mobilização de recursos humanos, financeiros e políticos (ESPÍNDOLA; RIBEIRO, 2020) e de liderança em pesquisa científica e participação em redes transnacionais de governos locais para as mudanças climáticas (RYAN, 2015).

Diante de um contexto em que os impactos das mudanças climáticas já são efetivos (PARMESAN; YOHE, 2003; IPCC, 2007), atingindo as populações e os territórios locais (KASPERSON et al., 2012), a adaptação climática é considerada a saída mais viável para enfrentar as mudanças climáticas, tendo em vista seus efeitos e benefícios no curto prazo e em escala local.

A adaptação climática é comumente integrada a outras estratégias já existentes, como o planejamento urbano, e não como uma nova proposta política (UITTENBROEK et al., 2014). 0 planejamento urbano consiste em uma política intersetorial essencial para lidar com as mudanças climáticas, particularmente na promoção da adaptação, uma vez que possibilita o desenvolvimento de políticas públicas ou ações de maneira integrada com outros departamentos setoriais. Por meio do planejamento urbano, enquanto política pública intersetorial, argumenta-se que os formuladores e gestores de políticas públicas podem elaborar e implementar medidas de enfrentamento das mudanças climáticas e, por consequência, promover benefícios à qualidade de vida das populações e ao meio ambiente na escala local (MAUAD, 2018).

No Brasil, onde mais de $80 \%$ das pessoas já vivem em áreas urbanas (IBGE, 2010), as legislações de planejamento urbano são estabelecidas, aprovadas e implementadas localmente, colocando, assim, os formuladores de políticas públicas e os planejadores urbanos como atores institucionais cruciais no processo de tomada de decisões políticas, especialmente voltadas para a adaptação climática. Para efeito deste estudo, investigam-se as cidades de Natal e de Curitiba, situadas em regiões macrogeográficas diferentes, com processos historicamente desordenados de urbanização que tendem a levar a impactos

\footnotetext{
${ }^{1}$ De acordo com o Departamento das Nações Unidas para Assuntos Econômicos e Sociais (UNDESA, 2019), aproximadamente 55\% da população do mundo vive em áreas urbanas desde 2019.
} 
climáticos severos e drásticos sobre as populações e o meio ambiente, em particular no contexto urbano.

Partindo da problematização de que a construção e a efetivação da capacidade de adaptação climática das cidades dependem de um modelo de gestão urbana local que priorize a integração do planejamento urbano e outros setores municipais com as questões climáticas, o objetivo deste artigo é analisar como as cidades de Natal e de Curitiba têm incorporado a questão climática, com enfoque especial para a adaptação, em suas agendas urbanas, compreendendo como o planejamento urbano pode contribuir para a inserção da capacidade adaptativa climática local. Para isso, a metodologia deste trabalho segue as orientações de uma abordagem de natureza qualitativa, utilizando um conjunto de instrumentos que permitem o alcance dos resultados.

Sob essa perspectiva, o artigo está estruturado em quatro seções, além desta introdução. A seguir, são detalhados os procedimentos metodológicos adotados com a pesquisa. Posteriormente, tem-se uma visão geral da literatura e da estrutura conceitual, que inclui adaptação, capacidade adaptativa e planejamento urbano na perspectiva das mudanças climáticas. Em seguida, identificam-se e analisam-se as estratégias climáticas, principalmente de adaptação, adotadas pelas cidades do campo empírico delimitado e são observadas as oportunidades e as barreiras enfrentadas pelas duas gestões para promover capacidade adaptativa climática. Por fim, conclui-se com as principais considerações sobre as análises realizadas no transcorrer do artigo.

\section{Metodologia}

A metodologia segue as orientações de uma pesquisa com abordagem de natureza qualitativa, conforme apresentado anteriormente. Com base em Deslauriers e Kérisit (2008), a escolha desse tipo de pesquisa se justifica, para efeito do presente estudo, por permitir que se investigue, de forma interpretativa e analítica, como a questão das mudanças climáticas é incorporada no âmbito das gestões urbanas de Natal e de Curitiba, de modo a compreender como o planejamento urbano pode contribuir para a construção e efetivação da capacidade adaptativa climática local.

A escolha de Natal e Curitiba se dá, primeiramente, em função de serem capitais de estados (Rio Grande do Norte e Paraná, respectivamente). Essas áreas, ainda que carentes de estudos interdisciplinares sobre mudanças climáticas e seus impactos nas cidades (BAI et al., 2018), são espaços com maior capacidade de mobilização de recursos humanos, financeiros e políticos quando comparados às médias e pequenas cidades (ESPÍNDOLA; RIBEIRO, 2020), servindo de "espelho" para essas últimas.

Não obstante, a seleção de Natal e Curitiba vai além desses aspectos. Outros dois motivos são: o primeiro é a representação de cidades com vulnerabilidade socioclimática alta (Natal) e baixa (Curitiba), com condições sociais, econômicas, ambientais e climáticas diferentes no contexto brasileiro (DARELA FILHO et al., 2016); e, o segundo, está relacionado 
com o fato de os planejamentos urbanos apresentarem trajetórias diferentes quanto à incorporação e integração das questões ambientais.

Para atingir o objetivo proposto, utiliza-se um conjunto de instrumentos de pesquisa. Inicialmente, realiza-se a pesquisa bibliográfica, por meio da busca de artigos científicos em indexadores como Portal de Periódicos Capes e Scopus sobre planejamento urbano e adaptação climática, visando subsidiar a revisão da literatura e a estrutura conceitual deste artigo.

A pesquisa caracteriza-se também como estudo de casos (YIN, 2010). Esse método possibilita observar se um planejamento urbano integrado à questão ambiental tem influência na capacidade de respostas de uma cidade aos efeitos das mudanças climáticas e, assim, na construção de um potencial de adaptação climática. Esse estudo de casos utiliza pesquisas documental e de campo, com aplicação de entrevistas semiestruturadas.

Na pesquisa documental, são analisadas as legislações, as políticas públicas, entre outras medidas que estão relacionadas com as questões urbanas e ambientais de Natal e de Curitiba. A pesquisa documental é considerada um importante instrumento de coleta de dados para esta análise, pois possibilita que sejam verificadas se as legislações, as políticas públicas ou outras iniciativas de caráter municipal fazem menção às mudanças climáticas, estabelecendo princípios, diretrizes e/ou objetivos para lidar com os desafios impostos localmente pelas mudanças climáticas.

De forma paralela, realiza-se a pesquisa de campo por meio da aplicação de entrevistas semiestruturadas com 12 interlocutores municipais que atuaram na gestão urbana do campo empírico delimitado entre agosto de 2017 e julho de 2018. Compreende-se que esses interlocutores são importantes para o presente estudo por serem atores-chave no processo de tomada de decisões políticas atrelado às mudanças climáticas locais.

É importante destacar que as seis primeiras entrevistas foram subsidiadas por meio do projeto de pesquisa nacional CiAdapta, ${ }^{2}$ enquanto as demais foram realizadas pelo primeiro autor deste artigo como forma de dar continuidade à pesquisa de campo de sua dissertação de Mestrado que, por sua vez, estava integrada ao projeto mencionado. 0 objetivo principal dessas entrevistas era coletar dados e informações sobre as estratégias urbanas conectadas com a adaptação climática e os elementos que influenciam a capacidade adaptativa climática das cidades. As entrevistas foram gravadas e, posteriormente, transcritas, mediante autorização prévia dos entrevistados e assinatura do Termo de Consentimento Livre e Esclarecido (TCLE).

Os dados coletados com a pesquisa documental e as entrevistas semiestruturadas são analisados a partir da técnica de análise de conteúdo (BARDIN, 2011), considerada de ampla validação em pesquisas qualitativas (MOZZATO; GRZYBOVSKI, 2011), auxiliando, assim, no desenvolvimento e alcance dos resultados deste estudo. Dentre os vários recortes da análise

\footnotetext{
${ }_{2}$ Finalizado em 2019, o projeto de pesquisa "Cidades, Vulnerabilidade e Mudanças Climáticas: uma abordagem integrada e interdisciplinar para análise de ações e de capacidade adaptativa" foi coordenado pela Profa. Dra. Gabriela Marques Di Giulio, da Universidade de São Paulo (USP). 0 objetivo era investigar o que seis cidades brasileiras têm adotado em termos de adaptação climática. Para mais detalhes, acessar: https://ciadapta.webnode.com/.
} 
de conteúdo (CAMPOS, 2004), optou-se pela análise temática, isto é, por temas a partir das unidades de análise (palavras, frases ou parágrafos), pois se entende que se trata da técnica que melhor permite 0 atendimento dos objetivos deste estudo e a realização de associações com os conceitos definidos no processo de revisão da literatura e estrutura conceitual.

Para Bardin (2011), a análise de conteúdo é organizada em três etapas principais, sendo a primeira a "pré-análise”, que consiste na seleção e organização do material obtido, neste caso, com a pesquisa documental e as entrevistas semiestruturadas. Em seguida, tem-se a segunda etapa, "exploração do material”, que permite a escolha e classificação das unidades de análise (BARDIN, 2011). É nessa fase que o material levantado é submetido à codificação. As categorias de análise desta pesquisa (Quadro 1) são, então, definidas considerand o o tema central. Essas categorias seguem a grade de análise mista, ou seja, ao passo que a coleta e a análise de dados são realizadas, outras categorias são inseridas, ainda que já se tenha uma grade inicial (BARDIN, 2011). A grade das oito primeiras categorias é inserida a partir da análise dos documentos e das entrevistas, enquanto a grade com as oito demais é incluída a partir do referencial teórico-conceitual (Quadro 1).

QUADRO 1

Categorias de análise da pesquisa

\begin{tabular}{|c|c|}
\hline Categorias analíticas & Descrição \\
\hline $\begin{array}{l}\text { Políticas municipais de mudanças } \\
\text { climáticas }\end{array}$ & $\begin{array}{l}\text { Existência de políticas públicas municipais que visam promover a } \\
\text { mitigação e/ou a adaptação }\end{array}$ \\
\hline Planos diretores municipais & Menção das mudanças climáticas nos planos diretores municipais \\
\hline $\begin{array}{l}\text { Comitês/fóruns locais de mudanças } \\
\text { climáticas }\end{array}$ & $\begin{array}{l}\text { Identificação da participação em comitês e/ou fóruns locais de } \\
\text { mudanças climáticas }\end{array}$ \\
\hline Infraestrutura verde & $\begin{array}{l}\text { Existência de iniciativas de infraestrutura verde alinhadas à adaptação } \\
\text { climática }\end{array}$ \\
\hline Drenagem urbana & $\begin{array}{l}\text { Alinhamento dos projetos e das obras de drenagem urbana com as } \\
\text { medidas adaptativas climáticas }\end{array}$ \\
\hline Mobilidade urbana & $\begin{array}{l}\text { Existência de estratégias de mobilidade urbana conectadas com a } \\
\text { adaptação climática }\end{array}$ \\
\hline $\begin{array}{l}\text { Gestão de vulnerabilidades e riscos } \\
\text { socioambientais }\end{array}$ & $\begin{array}{l}\text { Identificação de intervenções de redução de vulnerabilidades e riscos } \\
\text { associados aos impactos climáticos }\end{array}$ \\
\hline $\begin{array}{l}\text { Redes transnacionais de mudanças } \\
\text { climáticas }\end{array}$ & $\begin{array}{l}\text { Participação em redes subnacionais e transnacionais de governos } \\
\text { locais para as mudanças climáticas }\end{array}$ \\
\hline Acesso e uso das informações & Acesso e uso das informações técnicas e científicas sobre o clima \\
\hline $\begin{array}{l}\text { Recursos econômicos, institucionais e } \\
\text { tecnológicos }\end{array}$ & $\begin{array}{l}\text { Capacidade de alocação de recursos econômicos, institucionais e } \\
\text { tecnológicos para lidar com os efeitos das mudanças climáticas }\end{array}$ \\
\hline Adaptação sustentável & $\begin{array}{l}\text { Interseção entre adaptação, mitigação e desenvolvimento sustentável } \\
\text { a partir da experimentação de políticas públicas }\end{array}$ \\
\hline $\begin{array}{l}\text { Sinergias entre capacidades genéricas } \\
\text { e específicas }\end{array}$ & $\begin{array}{l}\text { Capacidade de combinação entre as necessidades básicas humanas e } \\
\text { as ferramentas necessárias para responder aos impactos climáticos }\end{array}$ \\
\hline Capital social & $\begin{array}{l}\text { Identificação de relações sociais informais e de compactação de } \\
\text { valores dos indivíduos de uma determinada comunidade }\end{array}$ \\
\hline Confiança & $\begin{array}{l}\text { Existência de relações de confiança entre os atores que fomentam a } \\
\text { adaptação climática }\end{array}$ \\
\hline Empreendedores de políticas públicas & Presença de empreendedores de políticas públicas \\
\hline $\begin{array}{l}\text { Processos de governança } \\
\text { participativos }\end{array}$ & $\begin{array}{l}\text { Identificação de processos de governança baseados em multiníveis e } \\
\text { multiatores, com enfoque especial para a participação da sociedade civil }\end{array}$ \\
\hline
\end{tabular}

Fonte: Elaboração própria a partir de Teixeira, Pessoa e Di Giulio (2020a; 2020b). 
A partir dessas categorias, os resultados são interpretados de forma reflexiva e crítica na terceira e última fase, "tratamento dos resultados - inferência e interpretação", com o intuito de torná-los significativos e válidos (BARDIN, 2011). Minayo (2001) acrescenta que a finalidade dessa etapa é confirmar se as hipóteses e os objetivos do trabalho em questão foram alcançados ou não, buscando a ampliação do conhecimento sobre o tema em estudo.

\section{Revisão da literatura e estrutura conceitual}

As cidades, como apresentado nas discussões introdutórias, são reconhecidas como cruciais no enfrentamento das mudanças climáticas (HUITEMA; BOASSON; BEUNEN, 2018), atuando por meio de duas frentes principais: a mitigação e a adaptação. Tendo em vista que a mitigação ${ }^{3}$ é pouco efetiva por não alterar os padrões de vida insustentáveis de produção e consumo de bens, nem mesmo modificar os modelos de gestão urbana (GIDDENS, 2010), e que os impactos das mudanças climáticas já são efetivos (PARMESAN; YOHE, 2003; IPCC, 2007), a adaptação é defendida neste estudo como a alternativa mais viável de enfrentamento das mudanças climáticas, com efeitos no curto prazo, beneficiando as populações e o meio ambiente no nível local, atingidos mais diretamente pelas mudanças climáticas.

Em termos conceituais, assume-se nesta discussão a adaptação climática como um processo de ajustamentos (de uma cidade, por exemplo), com o objetivo de antecipar os possíveis e potenciais impactos das mudanças climáticas no nível local, de modo a reduzir as múltiplas vulnerabilidades a situações de riscos socioambientais. Autores como Posey (2009) afirmam que a adaptação climática está intimamente associada à noção de capacidade adaptativa climática que, segundo Eakin, Lemos e Nelson (2014), consiste no potencial que as cidades têm em buscar respostas de adaptação climática, de maneira que assimilem os efeitos das mudanças climáticas.

Os aspectos (as variáveis) principais que influenciam direta ou indiretamente a capacidade de adaptação climática das cidades são sistematizados por estudos como o de Teixeira, Pessoa e Di Giulio (2020a): acesso e uso das informações; capacidade de alocação de recursos econômicos, institucionais e tecnológicos; intersecção entre adaptação, mitigação e desenvolvimento sustentável; sinergias entre capacidades genéricas e específicas; capital social; relações de confiança; presença de empreendedores de políticas públicas; e processos de governança participativos.

A capacidade de adaptação das cidades aos efeitos das mudanças climáticas envolve a integração entre os mais diversos setores municipais com domínios disciplinares sobre o clima, tais como gestão de recursos hídricos, mobilidade urbana e gestão de riscos de desastres (BARROS, 2017). Wamsler, Brink e Rivera (2013) acrescentam que o planejamento urbano é outro setor da gestão urbana local que necessita estar diretamente relacionado à adaptação climática, pois promove a regulação da ocupação e uso do solo das áreas

\footnotetext{
${ }^{3}$ Nesta análise, assume-se a mitigação enquanto o conjunto de estratégias que visam a redução ou eliminação das emissões globais de GEE (IPCC, 2007).
} 
urbanas, possibilitando a redução de vulnerabilidades e riscos associados às mudanças climáticas.

Com a gestão de redução e adaptação de riscos sendo uma temática transversal, os formuladores e planejadores das cidades necessitam compreender como a adaptação climática pode ser incorporada ou integrada nas práticas do planejamento urbano, ainda que o conhecimento relacionado a tais práticas seja escasso (GREIVING; FLEISCHHAUER, 2012).

Uittenbroek et al. (2014) assinalam que as estratégias de adaptação climática estão, geralmente, integradas a políticas públicas ou ações já existentes, como é o caso do próprio planejamento urbano, o que é denominado na literatura como abordagem de integração. Este tipo de abordagem é considerado mais viável por possibilitar custos mais baixos, na medida em que as respostas climáticas são associadas a outras que já existem (UITTENBROEK et al., 2014). Assim, a abordagem de integração é reconhecida como útil, com desempenho efetivo para enfrentar as mudanças climáticas, por criar efeitos sinérgicos, aumentar a eficiência e eficácia das respostas de adaptação climática, reduzir contradições entre as políticas existentes e evitar a competição entre adaptação e outras prioridades políticas locais (KOK; DE CONINCK, 2007; RUNHAAR et al., 2018).

O planejamento urbano, enquanto um instrumento de regulação e organização do espaço urbano por parte do poder público (neste caso, municipal), é amplamente reconhecido pelas comunidades científicas e pelos profissionais da gestão pública como um mecanismo essencial no contexto da adaptação climática, seja positivamente ou negativamente (MCCLURE; BAKER, 2018). Considerando o papel de protagonismo que as cidades têm frente às mudanças climáticas, Apollaro e Alvim (2017) apontam que o planejamento urbano se configura em um mecanismo importante para lidar com os efeitos desse fenômeno nos espaços urbanos, porque possibilita a indução de transformações sustentáveis nesses espaços, permitindo a inserção de novas atitudes e práticas de ocupação e uso do solo urbano. Essas novas atitudes e práticas alteram os estilos de vida das populações que vivem nos espaços urbanos, auxiliando no enfrentamento das mudanças climáticas (APOLLARO; ALVIM, 2017).

No campo da adaptação climática, o planejamento urbano pode assumir duas abordagens principais: a autônoma (ou reativa) e a planejada (ou pró-ativa) (SMIT; PILIFOSOVA, 2003; RIBEIRO et al., 2009; UN-HABITAT, 2014). Ainda que esta última abordagem seja a mais ideal (APOLLARO; ALVIM, 2017) por se entender que há a necessidade de evitar ou reduzir a ocorrência de eventos climáticos, a primeira ainda é a mais empregada, sendo particularmente de caráter reativo (age após o impacto climático já ter acontecido). Não obstante, salienta-se que essa via não é a mais eficaz e eficiente para enfrentar as mudanças climáticas, uma vez que seus efeitos já são efetivos em todo o mundo por meio, por exemplo, do aumento da temperatura, da intensificação da ocorrência de eventos climáticos extremos e do aumento do nível do mar, o que exige planejamento por parte das cidades e suas gestões locais. 
Nessa perspectiva, o planejamento urbano, ainda que funcione como meio crucial de contribuir com a adaptação climática das cidades, tem se mostrado como um desafio para a adaptação, já que existe uma resistência da incorporação de estratégias adaptativas no nível local tendo em vista o planejamento urbano ser um processo diretamente associado, por exemplo, com a regulamentação e os valores da terra (CHU; ANGUELOVSKI; ROBERTS, 2017). Os desafios, assim como as oportunidades para o processo de adaptação climática local, podem ser observados na prática a partir dos dois casos apresentados nesta análise.

\section{Análise e discussão dos resultados}

\section{Estratégias locais conectadas com as mudanças climáticas}

No âmbito de Natal, constata-se que a cidade não apresenta uma política municipal de mudanças climáticas, o que é reflexo, para Teixeira, Pessoa e Di Giulio (2020b), da falta de preocupação do planejamento urbano local com as questões ligadas ao meio ambiente e, sobretudo, ao clima. Segundo os autores, o plano diretor em vigência (de 2007) de Natal não traz levantamento de princípios, diretrizes e/ou objetivos relacionados às mudanças climáticas locais, tampouco à adaptação climática. Apesar disso, Natal apresenta a delimitação de dez Zonas de Proteção Ambiental (ZPAs), que funcionam como meios de frear a expansão urbana desordenada na cidade e seu transbordamento metropolitano.

Nesse aspecto, um bom exemplo pode ser observado na fala do representante da Secretaria Municipal de Mobilidade Urbana (STTU) de Natal: "A ausência de uma política local de mudanças climáticas ou de ações dentro do plano diretor de Natal faz com que a gestão municipal não possa tomar atitudes que tenham o intuito de evitar a ocorrência de desastres que acometem as populações" (Entrevistado 9, Secretaria Municipal de Mobilidade Urbana de Natal, 14 maio 2018). Estudos brasileiros recentes apontam que os planos diretores municipais não estão preocupados com a diminuição das emissões de GEE e, muito menos, com a adaptação aos efeitos locais das mudanças climáticas (SATHLER; PAIVA; BAPTISTA, 2019; ESPÍNDOLA; RIBEIRO, 2020).

Em situação diferente da de Natal, verifica-se que Curitiba incorpora a adaptação climática ao seu planejamento urbano, ao passo que, entre as iniciativas, aprovou em dezembro de 2020 o Plano de Adaptação e Mitigação das Mudanças Climáticas de Curitiba (PlanClima), apresentado como uma política alinhada a agendas internacionais como a Agenda 2030, da Organização das Nações Unidas (ONU) (CURITIBA, 2020). Em 2014, a referida política pública (que ainda estava em estágio de elaboração) passou a ser considerada uma das áreas estratégicas do atual plano diretor curitibano (CURITIBA, 2015), o qual, por sua vez, é mencionado pelo representante do Instituto de Pesquisa e Planejamento Urbano de Curitiba (IPPUC) como uma das iniciativas que a gestão de Curitiba tem desenvolvido para responder aos impactos climáticos: "o plano diretor [...] tem desenvolvido algumas estratégias para responder aos efeitos que as mudanças do clima vêm causando na cidade. 
Um bom exemplo é o plano municipal de mudanças climáticas" (Entrevistado 10, Instituto de Pesquisa e Planejamento Urbano de Curitiba, 6 jul. 2018).

Acrescenta-se ainda que o PlanClima foi idealizado em 2016 no âmbito do Fórum Curitiba sobre Mudanças Climáticas. Este Fórum, por seu turno, é um importante arranjo institucional de mudanças climáticas, atualizado recentemente pelo Decreto n. 572, cujo objetivo é "debater e propor medidas de mitigação e adaptação às inevitáveis mudanças climáticas para a cidade de Curitiba” (CURITIBA, 2019). Em Natal, constata-se que há o Comitê Municipal sobre Mudanças Climáticas e Ecoeconomia Sustentável, especificamente voltado para a mitigação das emissões de GEE (NATAL, 2009); todavia, não há relatos de continuidade de suas atividades, comprometendo a governança climática local, porque comitês/fóruns/redes locais de mudanças climáticas são cruciais para o planejamento urbano mais coerente com as questões ambientais (ICLEI; CEPS, 2013).

Embora o Plano e o Fórum citados de Curitiba não sejam dedicados exclusivamente a responder aos impactos já efetivos das mudanças climáticas, pontua-se que os mesmos são importantes estratégias de adaptação climática no âmbito local, uma vez que buscam integrar o reconhecimento das vulnerabilidades e dos riscos socioambientais da cidade.

É importante mencionar que, mais recentemente, a Prefeitura Municipal de Curitiba e o Serviço Nacional de Aprendizagem Industrial do Estado do Paraná (Senai/PR), por meio do Observatório Sistema Fiep, deram início a um projeto de construção do Painel de Indicadores de Mudança do Clima de Curitiba (CURITIBA, 2021). 0 projeto deverá ser implementado pela Prefeitura em parceria com o Sistema Fiep, o Google e o Local Governments for Sustainability (Iclei), visando subsidiar a tomada de decisões políticas e a implementação de políticas públicas climáticas (CURITIBA, 2021). Entende-se que um painel dessa natureza poderá auxiliar ainda mais no processo de adaptação climática local, pois possibilitará uma atualização periódica dos dados climáticos locais, colaborando com as decisões políticas e as ações a serem adotadas frente às mudanças climáticas no nível local.

Outro aspecto que se mostra diferente na análise de Natal e de Curitiba refere-se à existência de inventário local de emissões de GEE, presente em Curitiba, tendo sido o primeiro finalizado em 2011, com atualização em 2016 e, mais recentemente, em 2019. Já em Natal não existe uma iniciativa concreta nesse sentido.

Nesse ínterim, identifica-se que, em Curitiba, há um mapeamento das condições de vulnerabilidades socioambientais da cidade - "Avaliação de vulnerabilidade ambiental e socioeconômica para o município de Curitiba" -, o qual é atualizado periodicamente pela gestão urbana local e busca mapear não somente as vulnerabilidades socioambientais, mas também as potencialidades ambientais e socioeconômicas da cidade (IPPUC, 2014). Em Curitiba há também um mapeamento das áreas de riscos, com enfoque inicial para os riscos de inundações, denominado de "Mapa de riscos associados à inundação da bacia do rio Ponta Grossa - Curitiba/PR" e que serve de material-piloto para o mapeamento de outras áreas de riscos na cidade. 
Em Natal, diferentemente de Curitiba, há somente um mapeamento das áreas de riscos da cidade ("Plano municipal de redução de riscos de Natal"), elaborado sob a coordenação da Secretaria Municipal de Meio Ambiente e Urbanismo (Semurb) de Natal e que se encontra desatualizado desde 2008. Os estudos de vulnerabilidades e riscos socioambientais são de fundamental importância para os processos de formulação e implementação de políticas públicas ou ações de planejamento urbano, pois permitem que a gestão urbana seja capaz de promover estratégias que reduzam a ocorrência de eventos adversos (ROSENZWEIG; SOLECKI, 2010; MEDEIROS; SOUZA, 2016). Nessa perspectiva do gerenciamento dos riscos climáticos, observa-se que, tanto em Natal quanto em Curitiba, há planos de contingência. Em ambas as cidades, o referido Plano foi elaborado a partir da parceria entre a gestão urbana local e as instituições de pesquisa (como universidades federais).

Além dos estudos sobre vulnerabilidades e riscos socioambientais e dos planos de contingência de desastres, os sistemas de monitoramento e alertas de desastres são considerados por Eakin, Lemos e Nelson (2014) importantes iniciativas específicas de adaptação climática, que devem ser incorporadas ao planejamento urbano municipal, buscando reduzir as vulnerabilidades a situações de riscos socioambientais. Sobre isso, constata-se também que, em ambas as cidades, as gestões urbanas apresentam sistema de monitoramento e alertas de desastres, que se dá por meio de parceria com o Centro Nacional de Monitoramento e Alertas de Desastres Naturais (Cemaden), do Ministério da Ciência, Tecnologia, Inovações e Comunicações (MCTIC).

No âmbito dessa parceria, o sistema de monitoramento e alertas de desastres é constituído por estações pluviométricas que, por seu turno, possibilitam que a gestão urbana tenha acesso às informações climáticas. Em Natal, há ainda um radar meteorológico, instalado na Base Aérea de Natal (Bant). A rede de monitoramento e alertas de desastres é vista por Teixeira, Pessoa e Di Giulio (2020b) como crucial no contexto da adaptação climática, por se tratar de uma iniciativa que busca promover a redução de vulnerabilidades a situações de riscos socioambientais.

Em ambas as cidades, ainda que em estágios diferentes, identificam-se intervenções urbanas de infraestrutura verde. Em Natal, as ações dessa natureza se restringem à Lei n. 5.915/2009, que objetiva promover a recuperação e criação de novas áreas verdes em praças da cidade (NATAL, 2009). Já em Curitiba, existe uma gama de iniciativas que visam promover a adoção de novas áreas verdes públicas, bem como a manutenção das já existentes.

As obras de drenagem urbana também têm papel crucial no cenário de respostas aos efeitos das mudanças climáticas. No âmbito das cidades em análise, constata-se a existência de planos diretores de drenagem urbana que, por sua vez, regulam as intervenções físicas dessa natureza. A relação da drenagem urbana com a adaptação climática se dá na medida em que busca minimizar as situações de riscos de eventos adversos (TEIXEIRA; PESSOA; DI GIULIO, 2020b). 
Nas cidades estudadas, observa-se ainda a presença de iniciativas para a mobilidade urbana que, em ambas, são definidas no âmbito do plano diretor municipal. Todavia, tais ações apresentam estágios diferentes: em Natal, existe um Comitê Executivo para Coordenação Política do Plano de Mobilidade Urbana, definido por meio do Decreto n. 11.254 (NATAL, 2017), enquanto em Curitiba, ainda que seja dentro do atual plano diretor, verifica-se que não há prosseguimento nas decisões atreladas à política local de mobilidade urbana, regulamentada pela Lei n. 14.771 (CURITIBA, 2015). Geneletti e Zardo (2016) consideram que as intervenções urbanas voltadas para melhorar a mobilidade urbana são exemplos importantes de medidas de adaptação climática, auxiliando no alcance de resultados sustentáveis.

Ambas as cidades em análise integram o Iclei, rede global de governos locais e subnacionais preocupados com o desenvolvimento sustentável (ICLEI, s.d.). A integração das duas cidades a essa rede apresenta estágios diferentes, o que pode ser observado nas falas de dois representantes das Secretarias Municipais de Meio Ambiente de cada cidade: enquanto em Natal, "a integração da cidade ao Iclei [...] ainda tem pouca força e visibilidade” (Entrevistado 2, Secretaria Municipal de Meio Ambiente e Urbanismo de Natal, 22 ago. 2017), em Curitiba “a integração da cidade junto ao Iclei é forte e visível” (Entrevistado 4, Secretaria Municipal de Meio Ambiente de Curitiba, 17 set. 2017), participando de projetos em parceria com a rede, como o projeto Promovendo Estratégias de Desenvolvimento de Baixo Carbono em Economias Emergentes (Urban LEDS), financiado pela Comissão Europeia (ICLEI-BRASIL; PREFEITURA DE CURITIBA, 2016).

Além da adesão ao Iclei, Curitiba integra o Grupo C40 de Grandes Cidades para Liderança do Clima, que reúne cidades comprometidas em enfrentar as mudanças climáticas por meio da adoção de medidas de mitigação das emissões de GEE e de adaptação climática (C40 CITIES, s.d.). Essa adesão é corroborada na fala de outra representante da Secretaria Municipal de Meio Ambiente (SMMA) de Curitiba: "A gente tem, inclusive, assinado compromissos internacionais com o C40" (Entrevistado 12, Secretaria Municipal de Meio Ambiente de Curitiba, 22 jul. 2018).

Nesse sentido, em termos gerais, identifica-se que as cidades estudadas, principalmente Curitiba, apresentam algumas estratégias urbanas alinhadas às mudanças climáticas, ainda que enfrentem desafios e entraves para construir e efetivar sua capacidade adaptativa local. Esses desafios/entraves, bem como as possibilidades para a construção e efetivação dessa capacidade no contexto das cidades em estudo, são discutidos a seguir.

\section{Capacidade de adaptação climática no contexto de cidades}

A capacidade que as cidades têm em se adaptar às mudanças climáticas é influenciada direta ou indiretamente por um conjunto de variáveis (aspectos), conforme apresentado no momento de revisão da literatura deste artigo. A primeira dessas variáveis é o acesso e uso das informações técnicas e científicas sobre o clima. Para Carvalho e Furtado (2015), 
esse aspecto é importante, pois o processo de adaptação climática exige da gestão o reconhecimento da necessidade de se adaptar aos impactos das mudanças climáticas, além da compreensão e do conhecimento das opções de resposta disponíveis e quais, dentre estas, são as mais adequadas.

Sobre esse primeiro aspecto, salienta-se que, em Natal e Curitiba, os atores institucionais reconhecem que os impactos das mudanças climáticas estão em curso, bem como que o acesso e o uso de informações climáticas por meio, por exemplo, de centros especializados como o Cemaden auxiliam na antecipação desses impactos, contribuindo para a adaptação climática. Por outro lado, esses interlocutores apontam que há ainda a necessidade de novos estudos de vulnerabilidades e riscos socioambientais e até mesmo de atualização dos já existentes. Kirchhoff (2013) afirma que, em geral, o uso da informação científica pelas gestões ainda não é o desejado.

Outra variável refere-se à capacidade de recursos econômicos, institucionais e tecnológicos que, nos casos de Natal e de Curitiba, se apresenta de forma diferente. Nessa última cidade, observa-se que a gestão urbana, apesar de não ter uma rubrica específica para o tema das mudanças climáticas, "tem buscado financiar ações relacionadas ao tema através, por exemplo, de fundos como o Fundo Municipal do Meio Ambiente que financiou o primeiro inventário de emissões de efeito estufa do município" (Entrevistado 5, Secretaria Municipal de Meio Ambiente de Curitiba, 18 set. 2017).

Já em Natal, verifica-se que a gestão urbana local não tem procurado por financiamentos para as questões do clima urbano local, tampouco tem destinado investimento financeiro para a temática. Fankhauser e McDermott (2014) assinalam que essa falta de investimento no tema está relacionada com o déficit de adaptação (déficit entre o que se precisa e o que se sabe para a promoção da adaptação climática) que, por seu turno, perpassa pela ausência de recursos (econômicos, institucionais, tecnológicos, entre outros).

Um terceiro aspecto é a intersecção entre adaptação, mitigação e desenvolvimento sustentável pelo poder público local, a partir da experimentação de diferentes abordagens sociais e tecnológicas para lidar com as mudanças climáticas locais (DI GIULIO et al., 2017). Para estes autores, a experimentação de políticas públicas exerce um papel central nos processos de tomada de decisões sobre as mudanças climáticas, uma vez que promove a sustentabilidade nas cidades, contribuindo, por exemplo, para o alcance de ganhos econômicos e a redução de impactos ambientais associados às mudanças climáticas.

Especificamente no caso das cidades analisadas, destaca-se que, em Natal, a ausência de incorporação e efetivação do tema das mudanças climáticas na agenda governamental e urbana local se dá em função da falta de vontade política, que compromete a elaboração e a implementação de iniciativas climáticas. 0 representante do Departamento de Defesa Civil e Ações Preventivas de Natal descreve que "a vontade política deve desempenhar um papel menor em algumas políticas públicas, como uso e ocupação do solo, que transcendem mandatos políticos" (Entrevistado 1, Departamento de Defesa Civil e Ações Preventivas de Natal, 21 ago. 2017). 
Já em Curitiba, sinaliza-se para uma realidade diferente da de Natal, uma vez que as mudanças climáticas vêm se configurando, de certo modo, em temática prioritária na agenda governamental e urbana local, com a adoção e implementação de iniciativas climáticas urbanas, sejam de mitigação das emissões de GEE ou de adaptação climática. Essas medidas são concretizadas por meio de arranjos institucionais (CAMPOS; PHILIPPI JR.; SANTANA, 2015), como é o caso do Fórum Curitiba sobre Mudanças Climáticas.

A quarta variável que influencia a capacidade das cidades em promover processos de adaptação climática refere-se às sinergias entre capacidades genéricas e específicas. 0 investimento em ações de planejamento e redução de possíveis desastres é um exemplo da capacidade específica e que, quando esta é combinada com a capacidade genérica (produtividade econômica e níveis de redução, por exemplo), configura-se em outro aspecto que influencia na capacidade de uma cidade em se adaptar aos efeitos das mudanças climáticas (EAKIN; LEMOS; NELSON, 2014). Para estes autores, as sinergias entre capacidades genéricas e específicas são importantes para facilitar e promover capacidade adaptativa e gestão de riscos.

Esse aspecto se mostra como um desafio nas duas cidades analisadas, na medida em que ambas as gestões urbanas apresentam dificuldades em combinar as capacidades genéricas com as específicas. Por exemplo, Natal e Curitiba possuem dificuldades em atender às necessidades das comunidades locais, principalmente daquela sem condição de vulnerabilidade e situação de riscos, por carecerem de estudos científicos dessa natureza. 0 representante do IPPUC argumenta que "para se atender às necessidades das populações da cidade, a sociedade também precisa colaborar", mas observa que "a sociedade diz que o prefeito que precisa fazer algo para responder esse ou outro problema da cidade" (Entrevistado 10, Instituto de Pesquisa e Planejamento Urbano de Curitiba, 6 jul. 2018).

O capital social e a confiança são outros dois aspectos de fundamental importância para a adaptação climática, pois referem-se à capacidade que uma determinada comunidade tem em agir de maneira coletiva (ENGLE; LEMOS, 2010). 0 capital social é, de acordo com Pelling e High (2005), relativo às relações sociais informais e à compactação de valores dos indivíduos de uma determinada comunidade. Sobre isso, pontua-se que, em ambos os casos estudados com este artigo, existe certa dificuldade por parte da comunidade local em responder a situações de eventos adversos, como deslizamentos de terra.

Quanto às relações de confiança, que envolvem elementos racionais e emocionais (PARKER et al., 2008), observam-se cenários distintos nas duas cidades, visto que em Natal existe, em certa medida, confiança entre a academia (quem produz o conhecimento) e a gestão pública (quem coloca em prática esse conhecimento), enquanto em Curitiba não há uma boa relação entre segmentos, o que acaba gerando dificuldades nas respostas aos possíveis eventos adversos climáticos. Parker et al. (2008) assinalam que, quando não existe confiança entre a academia e a gestão pública, por exemplo, não há benefícios como confiança no processo de tomada das decisões políticas sobre as questões do clima. 
Uma sétima variável está relacionada com a presença de empreendedores de políticas públicas em criar novas iniciativas para responder às mudanças climáticas ou transformar as que já existem (UITTENBROEK et al., 2014). No contexto de Natal, esses empreendedores (que são, por exemplo, gestores e técnicos municipais) possuem dificuldade em efetivar essa variável em virtude de fatores como vontade política ou até mesmo falta de autoridade e jurisdição para tomar decisões políticas relacionadas ao clima urbano.

Em Curitiba, verifica-se que os gestores e técnicos urbanos municipais também apontam para limitações como a falta de autoridade e jurisdição, porém, diferentemente de Natal, indicam uma preocupação por parte dos políticos municipais em relação às questões ambientais e climáticas. Essa preocupação é uma estratégia política para alocação de recursos financeiros, por exemplo, mas não se pode deixar de considerar como um aspecto importante para a inserção e efetivação da questão das mudanças climáticas no planejamento urbano local.

Nesse ínterim, o representante da Secretaria Municipal de Planejamento e Administração (SMPA) de Curitiba afirma que a "vontade política existe, o prefeito tem investido muito em energias limpas, a gente tem contratado um profissional para fazer isso na cidade. Então, a parte política que, em algumas cidades não existe, na nossa existe muito forte" (Entrevistado 6, Secretaria Municipal de Planejamento e Administração de Curitiba, 22 set. 2017). A vontade política consiste em um dos facilitadores principais para a elaboração e implementação de iniciativas climáticas, porém, na prática, sua concretização não ocorre de maneira fácil e rápida, exigindo arranjos institucionais como convênios e comitês (CAMPOS; PHILIPPI JR.; SANTANA, 2015).

Por último, o oitavo aspecto que influencia a capacidade das cidades em se adaptar às mudanças climáticas corresponde aos processos de governança participativos que, para Guimarães e Martin (2011), são compreendidos como a coordenação entre os atores interdependentes, para lidar com as questões da ação coletiva e da cooperação. No caso das cidades estudadas, considera-se que, em ambas, a participação dos atores da sociedade civil local sobre as questões do clima é bastante fraca, sendo considerada prejudicial para os processos de tomada de decisões políticas sobre a temática. A respeito disso, em Curitiba, por exemplo, um representante da SMMA comenta que "a participação de atores da sociedade civil nas tomadas de decisões pode atrapalhar em duas situações: na falta de conhecimento técnico; e no excesso de críticas às ações criadas, executadas, aprimoradas pela gestão" (Entrevistado 4, Secretaria Municipal de Meio Ambiente de Curitiba, 17 set. 2017).

De maneira geral, observa-se que a inserção da adaptação climática, enquanto um mecanismo de incentivo à construção de uma sociedade sustentável, ainda não se configura em uma agenda estratégica para ambas as cidades analisadas, muito embora em Curitiba se tenha observado um planejamento urbano mais alinhado à promoção de uma agenda governamental que prioriza a questão das mudanças climáticas e a efetivação de uma capacidade adaptativa climática local, conforme ressaltado nas conclusões deste artigo. 


\section{Conclusões}

Internacionalmente, reforça-se que as cidades têm um papel crucial no enfrentamento das mudanças climáticas, particularmente a partir da adoção de estratégias de adaptação, tendo em vista que essas iniciativas possuem efeitos e benefícios no nível local. Diversos exemplos em todo o mundo apontam que as medidas adaptativas climáticas tendem a ser mais facilmente implementadas se integradas a outras já existentes (DI GIULIO et al., 2019) e, em Natal e Curitiba, não se observa um cenário diferente, uma vez que as estratégias de adaptação climática, que são identificadas nessas cidades, estão comumente associadas ao planejamento urbano e a outras políticas setoriais diretamente relacionadas (gestão de riscos de desastres, por exemplo), o que permite gerar benefícios sustentáveis.

No âmbito das cidades em análise, pontua-se que as respostas climáticas desenvolvidas pelos respectivos governos locais apresentam estágios distintos, reflexo direto de seus planejamentos urbanos que se mostram diferenciados no que concerne à incorporação e integração das questões ambientais. Nessa perspectiva, considera-se que as mudanças climáticas não são percebidas como um problema de relevância maior, embora os gestores e técnicos locais demonstrem, em certa medida, preocupação com a temática.

Em contrapartida, a gestão de Curitiba apresenta uma maior associação das questões climáticas locais com as urbanas. Nessa cidade, o planejamento urbano a partir, por exemplo, do plano diretor em vigor tem possibilitado algumas ações de prevenção e resposta aos impactos das mudanças climáticas. Apesar disso, é importante considerar que a cidade de Curitiba apresenta ainda uma série de desafios e entraves para antecipar os efeitos das mudanças climáticas, de modo a reduzir vulnerabilidades e riscos socioambientais que são persistentes na cidade.

Nesse viés, com base na experiência de Curitiba e de outras cidades, afirma-se que o planejamento urbano pode contribuir para o processo de adaptação climática, pois funciona como um instrumento estratégico que visa legislar e regular a ocupação e o uso do solo urbano, de modo a reduzir as múltiplas vulnerabilidades a situações de riscos socioambientais, construindo e/ou reforçando a resiliência socioambiental das cidades e, assim, promovendo a sustentabilidade urbana.

Dessa forma, afirma-se que o objetivo proposto na introdução é alcançado ao passo que se consegue, com este artigo, observar quais iniciativas, em particular de adaptação climática, têm sido incorporadas no âmbito das cidades de Natal e de Curitiba. Com isso, observa-se como o planejamento urbano local pode exercer um papel essencial sobre a adaptação climática e, assim, a construção e efetivação da capacidade adaptativa local frente às mudanças climáticas.

Esta análise é de suma importância acadêmica, porque contribui para o debate nacional sobre planejamento urbano e adaptação climática, ao identificar o que duas cidades brasileiras têm feito para responder às mudanças climáticas. 0 artigo é também um alerta para as gestões locais, sobretudo de Natal e de Curitiba, ao assumir que estas cidades 
precisam, e muito, incorporar e efetivar a questão climática em suas agendas governamentais. Pontua-se ainda que este estudo é socialmente importante, pois as deficiências frente às mudanças climáticas encontradas nas duas cidades têm reflexos diretos sobre as populações, em especial aquelas com baixo poder aquisitivo.

\section{Agradecimentos}

Os agradecimentos são, em especial, à Coordenação de Aperfeiçoamento de Pessoal de Nível Superior (Capes), pelo apoio financeiro de toda pesquisa de dissertação de Mestrado do primeiro autor, e ao projeto de pesquisa CiAdapta (Cidades, Vulnerabilidade e Mudanças Climáticas: uma abordagem integrada e interdisciplinar para análise e ações de capacidade adaptativa) (Proc. 446032/2015-8), por ter subsidiado parcialmente os resultados da dissertação e, consequentemente, deste artigo.

\section{Referências}

APOLLARO, C.; ALVIM, A. Estratégias e desafios do planejamento urbano para a adaptação de cidades frente à mudança climática. Revista Meio Ambiente e Sustentabilidade, v. 13, n. 6, p. 29-49, 2017.

AYLETT, A. Progress and challenges in the urban governance of climate change: results of a global survey. Cambridge: MIT, 2014.

BAI, X. et al. Six research priorities for cities and climate change. Nature Climate Change, v. 555, p. 23-25, 2018.

BARDIN, L. Análise de conteúdo. São Paulo: Edições 70, 2011.

BARROS, B. C. 0 planejamento urbano e territorial e a adaptação às mudanças climáticas: o caso das cidades de Nova York, Cidade do México, Bogotá e Rio de Janeiro. Dissertação (Mestrado) - Universidade do Estado do Rio de Janeiro, Rio de Janeiro, 2017.

BROTO, V. C. Urban governance and the politics of climate change. World Development, v. 93, p. 1-15, 2017.

C40 CITIES - Grupo C40 de Grandes Cidades para Liderança do Clima. The power of C40 cities. [s.d.]. Disponivel em: https://www.c40.org/cities. Acesso em: 5 dez. 2020.

CAMPOS, C. J. G. Método de análise de conteúdo: ferramenta para a análise de dados qualitativos no campo da saúde. Revista Brasileira de Enfermagem, v. 57, n. 5, p. 611-614, 2004.

CAMPOS, P. P. S.; PHILIPPI JR., A.; SANTANA, P. Gestão integrada de políticas climáticas e urbanas: uma proposta de avaliação legislativa em municípios da Região Metropolitana de São Paulo. Sustentabilidade em Debate, v. 6, n. 1, p. 119-137, 2015.

CARVALHO, S. A. D.; FURTADO, A. T. Os desafios da adaptação às mudanças climáticas globais. ClimaCom Cultura Científica: Pesquisa, Jornalismo e Arte, ano, 2, 2015.

CHU, E.; ANGUELOVSKI, I.; ROBERTS, D. Climate adaptation as strategic urbanism: assessing opportunities and uncertainties for equity and inclusive development in cities. Cities, v. 60, p. 378-387, 2017. 
CURITIBA. Lei n. 14.771, de 17 de dezembro de 2015. Dispõe sobre a revisão do plano diretor de Curitiba de acordo com o disposto no art. 40, § 3으, do estatuto da cidade, para orientação e controle do desenvolvimento integrado do município. Diário Oficial do Município de Curitiba, 2015. Disponivel em: https://leismunicipais.com.br/a/pr/c/curitiba/lei-ordinaria/2015/1477/14771/ lei-ordinaria-n-14771-2015-dispoe-sobre-a-revisao-do-plano-diretor-de-curitiba-de-acordo-como-disposto-no-art-40-3-do-estatuto-da-cidade-para-orientacao-e-controle-do-desenvolvimentointegrado-do-municipio. Acesso em: 5 nov. 2018.

CURITIBA. Decreto n. 572, de 2019. Atualiza a composição do Fórum Curitiba sobre Mudanças do Clima e designa os membros titulares e suplentes. Diário Oficial do Município de Curitiba, 2019. Disponível em: https://mid.curitiba.pr.gov.br/2020/00299439.pdf. Acesso em: 8 dez. 2018.

CURITIBA. Plano de Ação Climática de Curitiba começa a ser implementado em 2021. Prefeitura Municipal de Curitiba, 15 dez. 2020. Disponível em: https://www.curitiba.pr.gov.br/noticias/ plano-de-acao-climatica-de-curitiba-comeca-a-ser-implementado-em-2021/57425. Acesso em 10 maio 2021.

CURITIBA. Curitiba reúne ações para conter aumento da temperatura. Prefeitura Municipal de Curitiba, 17 mar. 2021. Disponível em: https://www.curitiba.pr.gov.br/noticias/curitiba-reuneacoes-para-conter-aumento-da-temperatura/58315. Acesso em: 10 maio 2021.

DARELA FILHO, J. P. et al. Socio-climatic hotspots in Brazil: how do changes driven by the new set of IPCC climatic projections affect their relevance for policy? Climatic Change, v. 136, n. 3-4, p. 413-425, 2016.

DESLAURIERS, J.; KÉRISIT, M. O delineamento de pesquisa qualitativa. In: POUPART, J. et al. A pesquisa qualitativa: enfoques epistemológicos e metodológicos. Petrópolis: Vozes, 2008. p. 127-53.

DI GIULIO, G. M. et al. Mudanças climáticas, riscos e adaptação na megacidade de São Paulo, Brasil. Sustentabilidade em Debate, v. 8, n. 2, p. 75-87, 2017.

DI GIULIO, G. M. et al. Extreme events, climate change and adaptation in the state of São Paulo. Ambiente \& Sociedade, v. 22, p. 1-20, 2019.

EAKIN, H. C.; LEMOS, M. C.; NELSON, D. R. Differentiating capacities as a means to sustainable climate change adaptation. Global Environmental Change, v. 27, p. 1-8, 2014.

ENGLE, N. L.; LEMOS, M. C. Unpacking governance: building adaptive capacity to climate change of river basins in Brazil. Global Environmental Change, v. 20, n. 1, p. 4-13, 2010.

ESPÍNDOLA, I. B.; RIBEIRO, W. C. Cities and climate change: challenges to Brazilian municipal Master Plans. Cadernos Metrópole, v. 22, n. 48, p. 365-396, 2020.

FANKHAUSER, S.; MCDERMOTT, T. K. Understanding the adaptation deficit: why are poor countries more vulnerable to climate events than rich countries? Global Environmental Change, v. 27, p. 9-18, 2014.

GENELETTI, D.; ZARDO, L. Ecosystem-based adaptation in cities: an analysis of European urban climate adaptation plans. Land Use Policy, v. 50, p. 38-47, 2016.

GIDDENS, A. A política da mudança climática. 1. ed. Rio de Janeiro: Zahar, 2010.

GREIVING, S.; FLEISCHHAUER, M. National climate change adaptation strategies of European states from a spatial planning and development perspective. European Planning Studies, v. 20, n. 1, p. 27-48, 2012.

GUIMARÃES, N. A.; MARTIN, S. Competitividade e desenvolvimento: atores e instituições locais. São Paulo: Editora Senac, 2001. 
HUITEMA, D.; BOASSON, E. L.; BEUNEN, R. Entrepreneurship in climate governance at the local and regional levels: concepts, methods, patterns, and effects. Regional Environmental Change, v. 18, p. 1247-1257, 2018.

IBGE - Instituto Brasileiro de Geografia e Estatística. Censo Demográfico 2010. Rio de Janeiro: IBGE, 2010. Disponível em: https://cidades.ibge.gov.br/brasil/pr/curitiba/panorama. Acesso em: 20 nov. 2020.

ICLEI - Local Governments for Sustainability. Conheça os governos que fazem parte da Rede na América do Sul. [s.d.]. Disponível em: http://sams.iclei.org/rede/associados-na-america-do-sul. html. Acesso em: 7 nov. 2018.

ICLEI - Local Governments for Sustainability; CEPS - Centre for European Policy Studies. Climate change adaptation: empowerment of local and regional authorities, with a focus on their involvement in monitoring and policy design. 2013. Disponivel em: https://cor.europa.eu/en/ documentation/studies/Documents/climate-change-adaptation.pdf. Acesso em: 18 out. 2018.

ICLEI-BRASIL; PREFEITURA DE CURITIBA. Curitiba - ações estratégicas: clima e resiliência. Curitiba, 2016. Disponível em: http://multimidia.curitiba.pr.gov.br/2016/00182811.pdf. Acesso em: 8 jan. 2019.

IPCC - Intergovernmental Panel on Climate Change. Summary for Policymakers. In: METZ, B.; DAVIDSON, O. R.; BOSCH, P. R.; DAVE, R.; MEYER, L. A. (ed.). Climate change 2007: mitigation. Contribution of Working Group III to the Fourth Assessment Report of the Intergovernmental Panel on Climate Change. Cambridge, UK and New York, NY: Cambridge University Press, 2007. Disponivel em: https://www.ipcc.ch/site/assets/uploads/2018/03/ar4_wg3_full_report-1.pdf. Acesso em: 5 nov. 2020.

IPPUC - Instituto de Pesquisa e Planejamento Urbano de Curitiba. Avaliação de vulnerabilidade ambiental e socioeconômica para o Município de Curitiba. São Paulo: ANTP, 2014. Disponível em: http://www.curitiba.pr.gov.br/conteudo/vulnerabilidade-ambiental-e-socioeconomica/2139. Acesso em: 5 nov. 2018.

KASPERSON, J. X. et al. Vulnerability to global environmental change. In: KASPERSON, J. X.; KASPERSON, R. E. The social contours of risk. Volume II: risk analysis, corporations and the globalization of risk. London: Earthscan, 2012. p. 245-285.

KIRCHHOFF, C. J. Understanding and enhancing climate information use in water management. Climatic Change, v. 119, n. 2, p. 495-509, 2013.

KOK, M. T. J.; DE CONINCK, H. C. Widening the scope of policies to address climate change: directions for mainstreaming. Environmental Science \& Policy, v. 10, n. 7-8, p. 587-599, 2007.

LECK, H.; ROBERTS, D. What lies beneath: understanding the invisible aspects of municipal climate change governance. Current Opinion in Environmental Sustainability, v. 13, p. 61-67, 2015.

MAUAD, A. C. E. Are we there yet? Cities and the IPCC responding to climate change. MundoramaRevista de Divulgação Científica em Relações Internacionais, 2018. Disponível em: file://C:/Users/NOTE/Downloads/ArewethereyetCitiesandthelPCCrespondingtoclimate changebyAnaCarolinaMauad.pdf. Acesso em: 29 abr. 2021.

MCCLURE, L.; BAKER, D. How do planners deal with barriers to climate change adaptation? A case study in Queensland, Australia. Landscape and Urban Planning, v. 173, p. 81-88, 2018.

MEDEIROS, C. N. de; SOUZA, M. J. N. de. Metodologia para mapeamento da vulnerabilidade socioambiental: caso do município de Caucaia, Estado do Ceará. Revista Eletrônica do PRODEMA, Fortaleza, v. 10, n. 1, p. 54-73, 2016. 
MINAYO, M. C. S. Pesquisa social: teoria, método e criatividade. Rio de Janeiro: Vozes, 2001.

MOZZATO, A. R.; GRZYBOVSKI, D. Análise de conteúdo como técnica de análise de dados qualitativos no campo da administração: potencial e desafios. Revista de Administração Contemporânea, v. 15, n. 4, p. 731-747, 2011.

NATAL. Decreto n. 8.841, de 18 de agosto de 2009. Cria o Comitê Municipal sobre Mudanças Climáticas e Ecoeconomia Sustentável. Diário Oficial do Município de Natal, 2009. Disponível em: https://www.natal.rn.gov.br/_anexos/publicacao/legislacao/decreto_8841.pdf. Acesso em: 14 jan. 2019.

NATAL. Decreto n. 11.254, de 25 de maio de 2017. Constitui Comissão Executiva de Coordenação Política do Plano de Mobilidade Urbana de Natal/RN. Diário Oficial do Município de Natal, 2017. Disponível em: https://leismunicipais.com.br/a/rn/n/natal/decreto/2017/1125/11254/decreton-11254-2017-constitui-comissao-executiva-de-coordenacao-politica-do-plano-de-mobilidadeurbana-de-natal-rn. Acesso em: 10 dez. 2018.

PARKER, S. et al. State of trust: how to build better relationships between councils and the public. London: Demos, 2008.

PARMESAN, C.; YOHE, G. A globally coherent fingerprint of climate change impacts across natural systems. Nature, v. 421, n. 6918, p. 37-42, 2003.

PELLING, M.; HIGH, C. Understanding adaptation: what can social capital offer assessments of adaptive capacity? Global Environmental Change, v. 15, n. 4, p. 308-319, 2005.

POSEY, J. The determinants of vulnerability and adaptive capacity at the municipal level: evidence from floodplain management programs in the United States. Global Environmental Change, v. 19, n. 4, p. 482-493, 2009.

RIBEIRO, M. et al. Design of guidelines for the elaboration of regional climate change adaptations strategies. Study for European Commission - DG Environment. Vienna: Ecologic Institute, 2009.

ROSENZWEIG, C.; SOLECKI, W. Introduction to climate change adaptation in New York City: building a risk management response. Annals of the New York Academy of Sciences, v. 1196, p. 13-18, 2010.

RUNHAAR, $H$. et al. Mainstreaming climate adaptation: taking stock about "what works" from empirical research worldwide. Regional Environmental Change, v. 18, n. 4, p. 1201-1210, 2018.

RYAN, D. From commitment to action: a literature review on climate policy implementation at city level. Climatic Change, v. 131, n. 4, p. 519-529, 2015.

SATHLER, D.; PAIVA, J. C.; BAPTISTA, S. Cidades e mudanças climáticas: planejamento urbano e governança ambiental nas sedes das principais regiões metropolitanas e regiões integradas de desenvolvimento. Caderno de Geografia, v. 29, n. 56, p. 262-262, 2019.

SMIT, B.; PILIFOSOVA, O. From adaptation to adaptive capacity and vulnerability reduction. In: SMITH, J. B.; KLEIN, R. J. T.; HUQ, S. Climate change, adaptive capacity and development. Londres: Imperial College Press, 2003. p. 9-28.

TEIXEIRA, R. L. P.; PESSOA, Z. S.; DI GIULIO, G. M. Mudanças climáticas e capacidade adaptativa no contexto da cidade do Natal/RN, Brasil. Revista Geotemas, v. 10, n. 1, p. 95-115, 2020 a.

TEIXEIRA, R. L. P.; PESSOA, Z. S.; DI GIULIO, G. M. Cidades, mudanças climáticas e adaptação: um estudo de caso de Natal/RN, Brasil. Desenvolvimento e Meio Ambiente, v. 54, p. 468-483, 2020b.

UITTENBROEK, C. J. et al. Political commitment in organising municipal responses to climate adaptation: the dedicated approach versus the mainstreaming approach. Environmental Politics, v. 23, n. 6, p. 1043-1063, 2014. 
UNDESA - United Nations Department of Economic and Social Affairs/Population Division. World urbanization prospects 2019. Nova York: United Nations, 2019. Disponível em: https:// population.un.org/wpp/. Acesso em: 20 de jun. de 2020.

UN-HABITAT - United Nations Human Settlements Programme. Planning for climate change: a strategic, value-based approach for urban planners. Nairobi: United Nations, 2014.

WAMSLER, C.; BRINK, E.; RIVERA, C. Planning for climate change in urban areas: from theory to practice. Journal of Cleaner Production, v. 50, p. 68-81, 2013.

YIN, R. K. Estudo de caso: planejamento e métodos. Trad. de Daniel Grassi. 2. ed. Porto Alegre: Bookman, 2010.

\title{
Sobre os autores
}

Rylanneive Leonardo Pontes Teixeira é doutorando e mestre em Estudos Urbanos e Regionais pela Universidade Federal do Rio Grande do Norte (UFRN) e graduado em Gestão de Políticas Públicas pela mesma instituição. Professor substituto do Departamento de Políticas Públicas da UFRN.

Zoraide Souza Pessoa é doutora em Ambiente e Sociedade pela Universidade Estadual de Campinas (Unicamp) e mestra em Ciências Sociais pela Universidade Federal do Rio Grande do Norte (UFRN). Professora associada do Departamento de Políticas Públicas e do Programa de Pós-Graduação em Estudos Urbanos e Regionais da UFRN.

\section{Endereço para correspondência}

Rylanneive Leonardo Pontes Teixeira

Rua do Reisado, 46, Lagoa Azul

59138-590 - Natal-RN, Brasil

Zoraide Souza Pessoa

Rua dos Tororós, 332, Alecrim

59032-550 - Natal-RN, Brasil

\begin{abstract}
Urban planning and climate adaptation: between possibilities and challenges in two major Brazilian cities
\end{abstract}

The aim of this paper is to investigate how the cities of Natal and Curitiba have incorporated the climate issue, with a special focus on adaptation, totheir urban agendas, understanding how urban planning can contribute to the insertion of local climate adaptive capacity. To that end, the methodology presents a qualitative approach, using bibliographic, documentary and field research, with the application of semi-structured interviews. The collected data are analyzed through content analysis. From the analysis and discussion of the results, we conclude that the incorporation of the climate issue, especially adaptation, in the planning and management of Natal and Curitiba still presents itself as a great and complex challenge.

Keywords: Climate changes. Adaptive capacity. Local governments. Urban sustainability. Brazil. 


\section{Resumen}

Planificación urbana y adaptación climática: entre posibilidades y desafíos en dos grandes ciudades brasileñas

El propósito de este artículo es investigar cómo las ciudades de Natal y Curitiba han incorporado el tema climático, con especial énfasis en la adaptación, en sus agendas urbanas, entendiendo cómo la planificación urbana puede contribuir con la inserción de la capacidad adaptativa climática local. Para eso, la metodología presenta un enfoque cualitativo que utiliza investigación bibliográfica, documental y de campo, con la aplicación de entrevistas semiestructuradas para lograr los resultados. Los datos recopilados son analizados por medio del análisis de contenido. Con el análisis y la discusión de los resultados se concluye que la incorporación del tema climático, especialmente la adaptación, en la planificación y gestión de Natal y Curitiba aún se presenta como un gran y complejo desafío.

Palabras clave: Cambio climático. Capacidad de adaptación. Gobiernos locales. Sostenibilidad urbana. Brasil.

Recebido para publicação em 21/01/2021 Aceito para publicação em 21/06/2021 